\title{
Sociodemographics, Social Vulnerabilities, and Health Factors Associated with Telemedicine Unreadiness Among US Adults
}

J Gen Intern Med 37(7):1811-3

DOI: $10.1007 / \mathrm{s} 11606-021-07051-6$

(C) This is a U.S. government work and not under copyright protection in the U.S.; foreign copyright protection may apply 2021

\section{BACKGROUND}

In the United States (US), the COVID-19 pandemic has caused substantial shifts in outpatient health care delivery from in-person visits to telemedicine. ${ }^{1}$ Although intended to maintain access to care, there is mounting concern that increasing use of telemedicine may widen the "digital divide" and exacerbate existing health inequities among at-risk populations. ${ }^{2}$

\section{OBJECTIVE}

To describe the prevalence and examine factors associated with telemedicine unreadiness among US adults.

\section{METHODS}

We performed a cross-sectional analysis (2016-2018) of the National Health Interview Survey (NHIS), a nationally representative telephone-based survey conducted by the Centers for Disease Control. We defined a survey participant as "telemedicine unready" if the participant reported all three of the following criteria: (1) lack of a computer; (2) lack of e-mail; and (3) lack of internet access. We extracted data on demographics, social vulnerabilities, and comorbid conditions from the survey. We defined social vulnerabilities as answering "yes" to any of the 39 survey questions assessing: (1) economic instability (10 questions), (2) disadvantaged neighborhood (4 questions), (3) low educational attainment (2 questions), (4) food insecurity (9 questions), and (5) social isolation (4 questions). We then estimated the national prevalence of all covariates. Finally, we examined the association of age, sex, race, ethnicity, health insurance, social vulnerabilities, and comorbidity with telemedicine unreadiness using multivariable logistic

Received March 30, 2021

Accepted July 15, 2021

Published online July 30, 2021 regression. NHIS data are publicly available and fully de-identified and not subject to institutional review board approval. All analyses took into account the complex survey design and incorporated person-level weights included in the NHIS datasets. ${ }^{3}$

\section{FINDINGS}

Among the 55,220 participants, over 1 in 6 (17.9\%) appear digitally unprepared to engage in telemedicine. Mean age was $47.5 \pm 18.1$ years, with sociodemographic characteristics representative of the USA. Respondents reported a mean of $2.1 \pm 1.2$ chronic conditions and $1.9 \pm 0.9$ social vulnerabilities.

Compared to the overall population, older aged individuals, racial and ethnic minorities (e.g., Blacks and Hispanics), and those with government-sponsored insurance (e.g., Medicare and Medicaid) or no insurance had a higher prevalence of telemedicine unreadiness. Additionally, those with multiple comorbid conditions and certain social vulnerabilities (lower educational attainment, food insecurity, and social isolation) had higher prevalence of telemedicine unreadiness (range: 20.3$36.3 \%)$. Notably, half (53\%) of all respondents $\geq 75$ years of age and those with dual Medicare and Medicaid coverage were telemedicine unready.

In adjusted analyses, advanced age (age 65-74: aOR 16.0, 95\% CI 13.6-18.9; age $\geq 75$ : aOR 56.3, 95\% CI 47.6-66.5) low educational attainment (aOR 6.4, 95\% CI 5.7-7.2), government-sponsored or no insurance (aOR range 2.6-5.2), minority background (Hispanic: aOR 2.9, 95\% CI 2.5-3.3; Black: aOR 1.7, 95\% CI 1.5-2.0; other: aOR 1.5, 95\% CI 1.21.9), food insecurity (aOR $2.2,95 \%$ CI $2.0-2.4$ ), and social isolation (aOR 1.5, 95\% CI 1.4-1.6) were associated with telemedicine unreadiness (Table 1).

\section{DISCUSSION}

In this nationally representative survey of US adults, over 1 in 6 appear to be digitally unprepared for telemedicine engagement with key vulnerable populations (e.g., older adults, racial and ethnic minorities, government-sponsored or no insurance, and socially vulnerable) at even higher risk. 
Table 1 National Prevalence and Odds of Telemedicine Unreadiness by Sociodemographics, Social Vulnerabilities, and Health Factors

\begin{tabular}{|c|c|c|}
\hline & \multicolumn{2}{|c|}{ Telemedicine unreadiness $*$} \\
\hline & $\begin{array}{l}\text { Prevalence, \% } \\
\text { (95\% CI) }\end{array}$ & $\begin{array}{l}\text { Adjusted odds } \\
\text { ratio (95\% CI) }\end{array}$ \\
\hline All respondents & $17.9(16.8-18.9)$ & - \\
\hline \multicolumn{3}{|l|}{ Age } \\
\hline $18-39$ & $5.2(4.7-5.7)$ & Reference \\
\hline $40-49$ & $10.4(9.4-11.4)$ & $3.0(2.6-3.5)$ \\
\hline $50-64$ & $16.4(15.4-17.5)$ & $6.5(5.6-7.6)$ \\
\hline $65-74$ & $26.4(25.0-27.8)$ & $16.0(13.6-18.9)$ \\
\hline$>75$ & $53.3(51.6-55.1)$ & $56.3(47.6-66.5)$ \\
\hline \multicolumn{3}{|l|}{ Sex } \\
\hline Male & $15.5(14.7-16.2)$ & Reference \\
\hline Female & $15.3(14.6-16.1)$ & $0.7(0.7-0.8)$ \\
\hline \multicolumn{3}{|l|}{ Race and ethnicity } \\
\hline White & $14.6(14.0-15.3)$ & Reference \\
\hline Black & $20.3(18.6-21.9)$ & $1.7(1.5-2.0)$ \\
\hline Other $\dagger$ & $19.2(15.9-22.4)$ & $1.5(1.2-1.9)$ \\
\hline Hispanic & $24.4(22.5-26.2)$ & $2.9(2.5-3.3)$ \\
\hline \multicolumn{3}{|l|}{ Health insurance } \\
\hline Private & $4.5(4.1-4.9)$ & Reference \\
\hline Medicare & $34.4(33.2-35.6)$ & $2.6(2.2-3.1)$ \\
\hline Medicaid & $23.2(21.4-25.0)$ & $3.9(3.3-4.6)$ \\
\hline Medicare and Medicaid & $57.0(53.3-60.6)$ & $5.2(4.2-6.5)$ \\
\hline Othert & $10.6(9.0-12.2)$ & $1.7(1.4-2.0)$ \\
\hline None & $21.6(19.7-23.5)$ & $3.6(3.1-4.1)$ \\
\hline \multicolumn{3}{|l|}{ Comorbidity count $\S$} \\
\hline 0 & $9.8(9.0-10.7)$ & Reference \\
\hline $1-2$ & $11.6(10.9-12.4)$ & $0.8(0.7-0.9)$ \\
\hline $3-4$ & $17.6(16.7-18.5)$ & $0.7(0.6-0.8)$ \\
\hline$\geq 5$ & $29.1(27.8-30.4)$ & $0.8(0.7-1.0)$ \\
\hline \multicolumn{3}{|l|}{ Social vulnerabilities } \\
\hline Economic instability & $15.9(15.2-16.7)$ & $1.0(0.9-1.0)$ \\
\hline Disadvantaged neighborhood & $17.4(16.3-18.6)$ & $1.1(1.0-1.2)$ \\
\hline Low educational attainment & $21.2(20.4-22.0)$ & $6.4(5.7-7.2)$ \\
\hline Food insecurity & $24.5(23.3-25.7)$ & $2.2(2.0-2.4)$ \\
\hline Social isolation & $20.2(19.3-21.0)$ & $1.5(1.4-1.6)$ \\
\hline
\end{tabular}

*Individuals met all three of the following criteria: (1) lack of a computer; (2) lack of e-mail; and (3) lack of internet access per survey responses

HIncludes American Indian, Native Hawaiian, Pacific Islander, other, do not know

Includes military health care, state-sponsored health plan, Indian Health Services, and single service plans (e.g., dental, vision, prescription)

SIncludes hypertension, hyperlipidemia, coronary artery disease, myocardial infarction, stroke, asthma, peptic ulcer disease, cancer, diabetes/prediabetes, chronic obstructive lung diseaselemphysema/ bronchitis, kidney disease, liver disease, arthritis/rheumatologic disease, migraine, chronic pain, obesity

Notes: Missingness ranged from 1.7 to 3.5\%. Missing data were not included in the analysis

While telemedicine has been shown to improve access for hard-to-reach populations, its use could further exacerbate existing health inequities. As health care systems contemplate increased and expanded post-pandemic use of telemedicine, these findings demonstrate that large segments of the population may not be prepared for such a change. If expansion does continue, healthcare systems will likely need to improve infrastructural support, such as access to high-speed internet and digital devices (i.e., computers and smartphones) to the most vulnerable populations. A recent nationwide initiative in the Veterans Health Administration to distribute video telehealth tablets to high-need patients with social and clinical access barriers may be one model moving forward. ${ }^{4}$

Our study has limitations. First, we lacked explicit data on smartphone access and use. Second, our findings may only apply to video-based visits as internet access is not needed for telephone-based telehealth visits. Finally, our analysis may lack current generalizability as it only focuses on prepandemic readiness which has potentially improved in the past year given the recent expansion of telemedicine.

In conclusion, there are striking disparities in telemedicine unreadiness among socially vulnerable and other at-risk populations - with as many as 1 in 2 being telemedicine unready in the highest risk populations. As health care systems continue to bolster telemedicine programs, attention to these disparities is urgently needed to improve health equity for the most vulnerable Americans.

Charlie M. Wray, DO, MS ${ }^{1,2,3}$

Janet Tang, $\mathrm{PhD}, \mathrm{MPH}^{1}$

Sachin Shah, MD, $\mathrm{MPH}^{1,4}$

Oanh Kieu Nguyen, MD, MAS ${ }^{1,5}$

Salomeh Keyhani, $M D, M P H^{1,6}$

${ }^{1}$ Department of Medicine, University of California, San Francisco,

San Francisco, USA

${ }^{2}$ Section of Hospital Medicine, San Francisco Veterans Affairs Medical Center,

San Francisco, CA, USA

${ }^{3}$ Section of Hospital Medicine, San Francisco VA Medical Center,

, CASan Francisco, USA

${ }^{4}$ Division of Hospital Medicine, University of California, San Francisco,

San Francisco, CA, USA

${ }^{5}$ Division of Hospital Medicine, San Francisco General Hospital,

San Francisco, USA

${ }^{6}$ Division of General Internal Medicine, San Francisco Veterans Affairs Medical Center,

San Francisco, USA

Corresponding Author: Charlie M. Wray, DO, MS; Section of Hospital Medicine, San Francisco VA Medical Center, , CASan Francisco, USA (e-mail: Charlie.Wray@ucsf.edu).

Funding Dr. Wray was supported by funds from a VA Health Services Research \& Development Career Development Award (CDA 19-349-3).

\section{Declarations:}

Conflict of Interest: The authors declare that they do not have a conflict of interest. 


\section{REFERENCES}

1. Koonin LM. Trends in the Use of Telehealth During the Emergence of the COVID-19 Pandemic — United States, January-March 2020. MMWR Morb Mortal Wkly Rep. 2020;69. doi:https://doi.org/10.15585/mmwr. mm6943a3

2. Ramsetty A, Adams C. Impact of the digital divide in the age of COVID-19. Journal of the American Medical Informatics Association. 2020;27(7):11471148. doi:https://doi.org/10.1093/jamia/ocaa078

3. NHIS - National Health Interview Survey. Published November 3, 2020 Accessed December 1, 2020. https://www.cdc.gov/nchs/nhis/index.htm
4. Zulman DM, Wong EP, Slightam C, et al. Making connections: nationwide implementation of video telehealth tablets to address access barriers in veterans. Jamia Open. 2019;2(3):323-329. doi:https://doi.org/10.1093/ jamiaopen/oozO24

Publisher's Note: Springer Nature remains neutral with regard to jurisdictional claims in published maps and institutional affiliations. 